\title{
Serum uric acid and risk of incident chronic kidney disease: a national cohort study and updated meta-analysis
}

Nianwei $\mathrm{Wu}^{1}$, Jing Xia ${ }^{1}$, Sen Chen ${ }^{1}$, Chuan $\mathrm{Yu}^{1}$, Ying $\mathrm{Xu}^{2}$, Chengfu $\mathrm{Xu}^{3}$, Tong Yan ${ }^{4}$, Ningxiu Li ${ }^{1}$, Yanjun Liu ${ }^{4,5^{*}}$ and Xiong-Fei $\operatorname{Pan}^{6,7,8^{*}}$ (1)

\begin{abstract}
Background: We prospectively examined the association between serum uric acid (SUA) levels and chronic kidney disease (CKD) in China and updated the evidence through a comprehensive meta-analysis of prospective studies worldwide.

Methods: Our original analyses were based on data from the China Health and Retirement Longitudinal Study. The primary exposure of interest was SUA at baseline, and the main outcome was incident CKD. Logistic regression models were used to examine the association between SUA levels and incident CKD. A meta-analysis was performed to pool our effect estimate and those from other cohort studies.

Results: During a 4-year follow-up, 180 participants developed incident CKD. Participants in the highest SUA quartile were 2.73 times as likely to develop incident CKD compared to those in the lowest quartile (multivariable-adjusted $\mathrm{OR}, 2.73 ; 95 \% \mathrm{Cl}, 1.65-4.50)$. Each $1 \mathrm{mg} / \mathrm{dL}$ increment in the SUA levels was associated with a $49 \%$ increased risk of incident CKD (multivariable-adjusted OR, 1.49; $95 \% \mathrm{Cl}, 1.28-1.74$ ). In the meta-analysis of 30 cohort studies (including the current study), pooled relative risks (95\% Cls) of incident CKD were $1.15(1.10-1.21)$ for SUA each $1 \mathrm{mg} / \mathrm{dL}$ increment, $1.22(1.14-1.30)$ for the highest versus lowest SUA group, and $1.17(1.12-1.23)$ for hyperuricemia versus no hyperuricemia.
\end{abstract}

Conclusions: Baseline SUA levels were associated with higher risk of incident CKD in middle-aged and elderly Chinese adults, and this positive association was confirmed in the meta-analysis of multiple cohort studies. Our findings may imply that SUA levels need to be routinely monitored for future CKD risk.

Keywords: Serum uric acid, Chronic kidney disease, Chinese, Cohort study, Meta-analysis

*Correspondence: liuyanjun_001@163.com; pxiongfei@gmail.com

${ }^{4}$ Center for Obesity and Metabolic Health, The Third People's Hospital of Chengdu \& The Affiliated Hospital of Southwest Jiaotong University, Chengdu, Sichuan, China

${ }^{6}$ Division of Epidemiology, Department of Medicine, Vanderbilt Epidemiology Center, Vanderbilt University Medical Center, Nashville, TN, USA

Full list of author information is available at the end of the article

\section{Background}

Chronic kidney disease (CKD) is a global public health challenge. The age-standardized prevalence rates of CKD were $8.7 \%$ worldwide and $7.2 \%$ in China in 2017 [1]. CKD is strongly associated with development of end-stage renal disease, cardiovascular events, and premature mortality [2, 3]. In addition, it compromises health-related quality of life and causes substantial financial burden for the patients [3]. It is thus essential to investigate potential modifiable risk factors and early interventions for CKD. original author(s) and the source, provide a link to the Creative Commons licence, and indicate if changes were made. The images or other third party material in this article are included in the article's Creative Commons licence, unless indicated otherwise in a credit line to the material. If material is not included in the article's Creative Commons licence and your intended use is not permitted by statutory regulation or exceeds the permitted use, you will need to obtain permission directly from the copyright holder. To view a copy of this licence, visit http://creativecommons.org/licenses/by/4.0/. The Creative Commons Public Domain Dedication waiver (http://creativeco mmons.org/publicdomain/zero/1.0/) applies to the data made available in this article, unless otherwise stated in a credit line to the data. 
Serum uric acid (SUA), an end-product of the metabolism of purine nucleotides, is commonly elevated in patients with CKD [4]. Although high SUA levels could be due to impaired clearance of uric acid in CKD, it is long speculated that high SUA levels may be implicated in the development and progression of CKD [5]. A systematic review of 15 cohort studies among 99,205 participants and 3,492 incident CKD cases showed a risk increase of $22 \%$ for each increment of $1 \mathrm{mg} / \mathrm{dL}$ SUA [6]. Of these included studies, four studies were conducted in China: three in health care settings in Chinese Taiwan [7-9] and one in a community setting in Chinese mainland [10]. Besides the restricted health care settings that may affect the generalizability, results from these studies were not very consistent. For example, of the four studies in Chinese populations, two in health care settings reported borderline or null associations of CKD with each unit of SUA increase in Chinese Taiwan [7, 9]. In addition, the positive association between SUA levels and CKD seemed to be stronger in Asians versus non-Asians, and in health-check populations versus non-health-check populations, despite a lack of statistical significance for the heterogeneity [6]. In this context, we hypothesized that SUA could be a risk factor for CKD in Chinese and proposed to test this hypothesis in a population-based cohort study with a large sample size in mainland China.

To this end, we conducted analyses using data from the China Health and Retirement Longitudinal Study (CHARLS), a national population-based longitudinal study, to assess the association between baseline SUA levels and risk of incident CKD. In addition, we conducted a comprehensive meta-analysis of findings from literature to corroborate our work in the Chinese population and update the evidence on this topic.

\section{Methods}

\section{Study design}

The CHARLS is an ongoing nationally representative study among adults aged 45 years or older that aims to assess aging-related issues in China. Details of the study design, survey methods, and procedures were described elsewhere [11]. Briefly, a multi-stage probability sampling procedure was performed to recruit 17,708 participants from 450 communities of 28 provinces at baseline (2011-2012). Trained staff collected data using faceto-face questionnaires via computer-assisted personal interviews, physical measurements, and blood samples for biomarker measurements at baseline and during the follow-up until 2015-2016. While the original CHARLS did not specifically address the etiology of kidney diseases, we utilized data on SUA and covariates at baseline and CKD outcomes during the follow-up to examine the association of SUA and risk of incident CKD. The original
CHARLS was approved by the Biomedical Ethics Review Committee of Peking University (IRB00001052-11,015), and all participants provided informed consent.

The eligibility of study participants and the study flow are presented in Additional file 1: Figure S1. Of the 17,708 study participants in CHARLS, we excluded 7,081 participants without blood samples and 496 participants who did not complete questionnaires at baseline. Of the remaining 10,131 participants, we excluded the participants who lacked information on SUA $(n=163)$ and CKD $(n=247)$ at baseline.

We also excluded the participants whose baseline estimated glomerular filtration rate (eGFR) was $<60 \mathrm{~mL} /$ $\min / 1.73 \mathrm{~m}^{2}(\mathrm{n}=114)$, who used medications for kidney diseases $(n=313)$, or who had prevalent malignancies $(\mathrm{n}=51)$, cardiovascular diseases (i.e., heart disease and stroke) $(n=1,565)$. Of the 7,678 eligible participants, we excluded the 2,188 participants who were lost to follow-up and 165 participants who lacked information on CKD in 2015-2016. 779 participants were additionally excluded due to data missing for age $(n=6)$, sex $(n=6)$, education level $(n=6)$, residence $(n=7)$, alcohol consumption $(\mathrm{n}=47)$, body mass index (BMI, $\mathrm{n}=679)$, and systolic blood pressure $(B P ; n=28)$ at baseline. 4,546 participants were included in final analysis. The 3,132 eligible participants who were excluded were more likely to be older, from urban areas, and to suffer from hypertension or diabetes, but were less likely to be never smokers, compared to the included 4,546 participants $(\mathrm{P} \leq 0.020$ for all, Additional file 1: Table S1).

\section{Study exposure and outcome}

The primary exposure was SUA at baseline, which was measured in the Youanmen Center for Clinical Laboratory of Capital Medical University using the SUA Plus method. We categorized SUA levels into quartiles $(<3.5$, 3.6-4.2, 4.3-5.0, and $>5.1 \mathrm{mg} / \mathrm{dL}$ ). Hyperuricemia was defined as a level of SUA $\geq 7.0 \mathrm{mg} / \mathrm{dL}$ in men or $\geq 6.0 \mathrm{mg} /$ $\mathrm{dL}$ in women [12].

The research outcome, incident CKD, was primarily defined as eGFR $<60 \mathrm{~mL} / \mathrm{min} / 1.73 \mathrm{~m}^{2}$ in $2015-2016$. The eGFR was calculated using the Chronic Kidney Disease Epidemiology Collaboration (CKD-EPI) Study equation: GFR = $141 \times \min (\mathrm{Scr} / \kappa, 1)^{\alpha} \times \max (\mathrm{Scr} / \kappa, 1)^{-1.209} \times 0.993^{\text {Age }}$

$\times 1.108$ [if female] $\times 1.159$ [if black], where $\mathrm{Scr}$ is serum creatinine, $\kappa$ is 0.7 for females and 0.9 for males, $\alpha$ is 0.329 for females and -0.411 for males, min means the minimum of $\mathrm{Scr} / \kappa$ or 1 , and max means the maximum of $\mathrm{Scr} / \kappa$ or 1 [13]. In a sensitivity analysis, we used the Modification of Diet in Renal Disease (MDRD) Study equation [14] to alternatively estimate eGFR: eGFR= $175 \times(S c r)^{-1.154} \times(a g e)^{-0.203} \times 0.742$ [if female] $\times 1.212$ [if black], 
where Scr is serum creatinine. Serum creatinine was measured by the rate-blanked and compensated Jaffe creatinine method.

\section{Covariate measurements}

Major covariates including sociodemographic characteristics, health-related lifestyle and behaviors, medical conditions, and anthropometrics were collected at baseline (2011-2012). Sociodemographic characteristics comprised age, sex (men and women), educational level (illiterate, primary school, and middle school or above) and residence (rural and urban). Health-related lifestyle and behaviors comprised cigarette smoking (current, former, and never), and alcohol drinking (current, former, and never). Current smoking was defined as having the habit of smoking cigarettes, cigars, tobacco, or pipe currently, and current drinking was defined as having drunk any alcoholic beverages (wine, beer, or liquor) for more than once a month in the past year. Medical conditions included prevalent hypertension, diabetes mellitus, and dyslipidemia (yes or no). Body weight and height were measured using a digital weight scale and stadiometer. BMI was defined as the body weight in kilograms divided by the square of the height in meters $\left(\mathrm{kg} / \mathrm{m}^{2}\right)$. BP was measured three times using an electronic blood pressure monitor (Omron HEM-7112; Omron [Dalian] Co, Dalian, China) on the right upper arm after 5 min of seated rest. Blood samples were collected from study participants by trained staff based on a standard protocol for biomarker measurements. Fasting blood glucose (FBG), triglyceride (TG), high-density lipoprotein cholesterol (HDL-C), lowdensity lipoprotein cholesterol (LDL-C), and total cholesterol (TC) were measured by the enzymatic colorimetric test. Glycosylated hemoglobin (HbA1c) was measured through the high performance liquid chromatography. Hypertension was defined as systolic $\mathrm{BP} \geq 140 \mathrm{mmHg}$ or diastolic $\mathrm{BP} \geq 90 \mathrm{mmHg}$ or self-reported physician diagnosis [15]. Prevalent diabetes was determined based on fasting blood glucose $\geq 126 \mathrm{mg} / \mathrm{dL}, \mathrm{HbA} 1 \mathrm{c} \geq 6.5 \%$, or self-reported physician diagnosis [16]. Dyslipidemia was defined as either or a combination of $\mathrm{TC} \geq 240 \mathrm{mg} / \mathrm{dL}$, $\mathrm{LDL}-\mathrm{C} \geq 160 \mathrm{mg} / \mathrm{dL}, \mathrm{HDL}-\mathrm{C}<40 \mathrm{mg} / \mathrm{dL}, \mathrm{TG} \geq 200 \mathrm{mg} /$ $\mathrm{dL}$, or self-reported physician diagnosis, according to the 2007 Chinese guidelines on the prevention and treatment of dyslipidemia [17].

\section{Statistical analyses}

Continuous variables were summarized as mean and standard deviation (SD), while categorical variables were presented as frequency and percentage. Baseline characteristics were compared using ANOVA (for continuous variables) or Pearson's chi-square (for categorical variables) tests across SUA quartiles.

Logistic regression models were employed to assess the relationship between baseline SUA and incident CKD with adjustments for a priori identified covariates in a stepwise manner. Odds ratios (ORs) with corresponding 95\% CIs were estimated. In Model 1, we adjusted for age (continuous, years), sex (male and female), residence area (rural and urban), and education level (illiterate, primary school, and middle school or above). In Model 2, we adjusted for variables in Model 1 plus BMI (continuous, $\mathrm{kg} / \mathrm{m}^{2}$ ), smoking status (never, former, and current), and alcohol consumption (never, former, and current). In Model 3, we adjusted for variables in Model 2 plus systolic BP (continuous, $\mathrm{mmHg}$ ), TC (continuous, $\mathrm{mg} / \mathrm{dL}$ ), TG (continuous, $\mathrm{mg} / \mathrm{dL}$ ), and prevalent diabetes mellitus (yes and no). In all these models, SUA was assessed as either a categorical variable (dichotomous or in quartiles) or a continuous variable (each $1 \mathrm{mg} / \mathrm{dL}$ ). Linear trends across SUA quartile groups were estimated by modeling SUA as the median of each quartile. In addition, subgroup analyses were conducted by sex (men and women), age ( $<60$ and $\geq 60$ years), education level (illiterate, primary school, and middle school or above), residence (rural and urban), BMI $\left(<24,24.0-27.9\right.$, and $\geq 28 \mathrm{~kg} / \mathrm{m}^{2}$ based on the Chinese criteria for overweight and obesity) [18], smoking status (current, former, and never), alcohol consumption (current, former, and never), hypertension (yes and no), prevalent diabetes mellitus (yes and no), and dyslipidemia (yes and no), and potential effect modifications (interactions) were explored by adding a product term of the stratifying variable and SUA to the final model and examined using a likelihood ratio test.

Four sensitivity analyses were conducted. First, we adjusted for prevalent dyslipidemia (yes and no), and hypertension (yes and no) as binary variables instead of continuous variables in the final model. Second, we conducted multiple imputations for 779 participants with missing covariates followed by logistic regression modeling. Under the assumption that data were missing at random, missing values were replaced by imputed ones from ten duplicate datasets that were created to reduce sampling variability from the imputation simulation, and estimates from the ten imputed datasets were combined to obtain the effect estimates and 95\% CI. Third, eGFR was estimated using the MDRD Study equation prior to CKD ascertainment. Fourth, we estimated the time-mean SUA as the arithmetic mean of the baseline (2011-2012) and follow-up (2015-2016) measurements, and modelled this variable as in the main analyses. All analyses were 
conducted using Stata 16.0 (Stata Corp LLC, College Station, Texas, US). $P<0.05$ was defined as statistical significance.

\section{Meta-analysis of cohort studies}

We conducted a meta-analysis of data from our study and other cohort studies that assessed the relationship between the SUA levels and incident CKD in adults. A literature search was performed using Medline (1948 to present) and Embase (1974 to present) for published studies up to July 30, 2021. We combined MeSH terms and keywords for "uric acid", "hyperuricemia", "chronic kidney disease", and "chronic renal insufficiency" in our search strategy. Records were screened by two investigators (NW and JX) independently with the following eligibility criteria: (1) cohort studies; (2) assessment of the association between SUA and new-onset CKD; and (3) reporting effect estimates and corresponding 95\% CIs such as hazard ratio (HR) or OR. Study-specific effect estimates were pooled using a random-effects model ( $Q$ test's $P<0.01$ ) or fixed-effects model (when $Q$ test's $P>0.01$ ). When studies reported effect estimates for men and women separately, they were pooled in individual studies by using random-effects meta-analysis, which were used to combine with those in other studies for the final meta-analysis. If effect estimates were reported as ORs, they were converted to relative risks (RRs) using the formula $(\mathrm{RR}=\mathrm{OR} /([1-\mathrm{pRef}]+[\mathrm{pRef} \times \mathrm{OR}])$, where pRef is the prevalence of the outcome in the reference group. HRs were regarded as approximate RRs for the meta-analysis. Subgroup analyses were conducted by major study characteristics including sex (men and women), mean age $(<60$ and $\geq 60$ years), population (Asian and non-Asian), and average follow-up $(<4.6$ and $\geq 4.6$ years, using the median of average follow-up durations across studies as the cut-off). Meta-analyses were conducted using Stata 16.0 (Stata Corp LLC, College Station, Texas, US). $P<0.05$ was defined as statistical significance.

\section{Results}

\section{Baseline characteristics}

Of 4,546 participants, the mean age was 58.57 (SD, 8.67) years, and 2,099 (46.2\%) were men (Table 1). Baseline mean (SD) statistics were $4.34(1.20) \mathrm{mg} / \mathrm{dL}$ for SUA, 0.76 (0.16) $\mathrm{mg} / \mathrm{dL}$ for serum creatinine, and $94.15(12.53) \mathrm{mL} /$ min per $1.73 \mathrm{~m}^{2}$ for eGFR. Participants with higher SUA were more likely to be older, men, urban residents, current/former smokers, or current alcohol drinkers, and to have hypertension and dyslipidemia. In addition, BMI, systolic BP, diastolic BP, TG, TC and serum creatinine levels increased with SUA levels, while HDL cholesterol decreased with SUA levels.

\section{Association between SUA levels and risk of incident CKD}

During the 4-year follow-up period, 180 participants developed incident CKD (Table 2). Despite a lack of statistical significance, hyperuricemia seemed to be positively associated with incident CKD (multivariable OR, 1.73; 95\% CI, 0.98-3.06). Participants in the fourth SUA quartile were 2.73 times as likely to develop incident CKD compared to those in the first quartile (OR, 2.73; 95\% CI, 1.65-4.50; Table 2). A linear trend was noted for the association between SUA quartiles and incident CKD $(P$ for trend $<0.001$ ). In addition, each $1 \mathrm{mg} / \mathrm{dL}$ increment in the SUA levels was associated with a $49 \%$ increased risk of incident CKD after adjustment for potential confounders (OR, 1.49; 95\% CI, 1.28-1.74) (Table 2). When prevalent dyslipidemia and hypertension were adjusted for instead of continuous measurements or when multiple imputations were employed for variables with data missing, there were no appreciable changes in the effect estimates (Additional file 1: Table S2-3). When we used the MDRD study equation to estimate GFR for CKD ascertainment, elevated SUA levels showed stronger positive associations with risk of incident CKD (OR, 3.18; 95\% CI, 2.01-5.03 for Quartile 4 versus 1; OR, 1.52; 95\% CI, $1.32-1.75$ for each $1 \mathrm{mg} / \mathrm{dL}$ increment; Additional file 1: Table S4). In the sensitivity analysis using time-mean SUA as the exposure, effect estimates were stronger than those from the main analyses (OR, 4.16; 95\% CI, 2.516.90 for Quartile 4 versus 1; OR, 1.97; 95\% CI, 1.69-2.30 for each $1 \mathrm{mg} / \mathrm{dL}$ increment; Additional file 1: Table S5). Our exploratory subgroup analyses found consistent positive associations across all subgroups $(P$ for interaction $\geq 0.167$ for all, Additional file 1: Figure S2).

\section{Meta-analysis for associations between SUA levels and incident CKD}

A total of 6,135 records were retrieved from two databases. After the exclusion of records that did not meet eligibility criteria (Additional file 1: Figure S3), a total of 30 studies (including the current study) were included in the meta-analysis (Additional file 1: Table S6). These studies comprised 577,334 participants and 35,980 incident cases of CKD (average follow-up durations: 4.6 years). 19 studies reported effect estimates for SUA as a continuous variable, 18 studies for SUA in quartiles, and 5 studies for SUA as a dichotomous variable. Pooled RRs (95\% CIs) in relation to incident CKD were 1.15 (1.10-1.21) for SUA each $1 \mathrm{mg} / \mathrm{dL}$ increment, 1.22 (1.14-1.30) for the fourth versus first SUA quartile, and 1.17 (1.12-1.23) for hyperuricemia versus no hyperuricemia (Fig. 1). We found no evidence of heterogeneity in subgroups by sex, age, population, and follow-up duration $(P \geq 0.112$ for all; Fig. 2). 
Table 1 Baseline characteristics of participants across SUA quartiles $(n=4,546)$

\begin{tabular}{|c|c|c|c|c|c|c|}
\hline \multirow[t]{3}{*}{ Characteristics } & \multicolumn{5}{|c|}{ SUA (mg/dL) } & \multirow[t]{3}{*}{$P$-value* } \\
\hline & Total & Quartile 1 & Quartile 2 & Quartile 3 & Quartile 4 & \\
\hline & & $(<3.5)$ & $(3.6-4.2)$ & $(4.3-5.0)$ & $(>5.1)$ & \\
\hline Men, n (\%) & 2099 (46.2\%) & $251(21.5 \%)$ & $420(37.1 \%)$ & $633(55.0 \%)$ & 795 (72.4\%) & $<0.001$ \\
\hline Education level, n (\%) & & & & & & $<0.001$ \\
\hline Illiterate & $1310(28.8 \%)$ & $428(36.7 \%)$ & $364(32.1 \%)$ & $310(27.0 \%)$ & $208(18.9 \%)$ & \\
\hline Primary school & $1882(41.4 \%)$ & $438(37.6 \%)$ & $468(41.3 \%)$ & $459(39.9 \%)$ & $517(47.1 \%)$ & \\
\hline Middle school or above & 1354 (29.8\%) & $299(25.7 \%)$ & $301(18.4 \%)$ & $381(33.1 \%)$ & $373(34.0 \%)$ & \\
\hline Urban residence, n (\%) & $626(13.8 \%)$ & $135(11.6 \%)$ & $145(12.8 \%)$ & $160(13.9 \%)$ & $186(16.9 \%)$ & 0.002 \\
\hline Smoking status, n (\%) & & & & & & $<0.001$ \\
\hline Current & $1388(30.5 \%)$ & $220(18.9 \%)$ & $292(25.8 \%)$ & $410(35.7 \%)$ & $466(42.4 \%)$ & \\
\hline Former & $359(7.9 \%)$ & $47(4.0 \%)$ & $67(5.9 \%)$ & $92(8.0 \%)$ & $153(13.9 \%)$ & \\
\hline Never & $2799(61.6 \%)$ & $898(77.1 \%)$ & $774(68.3 \%)$ & $648(56.3 \%)$ & $479(43.6 \%)$ & \\
\hline Alcohol consumption, n (\%) & & & & & & $<0.001$ \\
\hline Current & $1202(26.4 \%)$ & $170(14.6 \%)$ & $239(21.1 \%)$ & $328(28.5 \%)$ & $465(42.3 \%)$ & \\
\hline Former & $368(8.1 \%)$ & $77(6.6 \%)$ & $94(8.3 \%)$ & $109(9.5 \%)$ & $88(8.0 \%)$ & \\
\hline Never & $2976(65.5 \%)$ & $918(78.8 \%)$ & $800(70.6 \%)$ & $713(62.0 \%)$ & $545(49.6 \%)$ & \\
\hline Hypertension, n (\%) & $1171(25.8 \%)$ & $224(19.2 \%)$ & $255(22.5 \%)$ & $319(27.7 \%)$ & $373(34.0 \%)$ & $<0.001$ \\
\hline Prevalent diabetes mellitus, $\mathrm{n}(\%)$ & $671(14.8 \%)$ & $169(14.5 \%)$ & $151(13.3 \%)$ & $182(15.8 \%)$ & $169(15.4 \%)$ & 0.348 \\
\hline Dyslipidemia, n (\%) & $1879(41.3 \%)$ & $392(33.6 \%)$ & $440(38.8 \%)$ & $525(45.7 \%)$ & $522(47.5 \%)$ & $<0.001$ \\
\hline Age, mean (SD), yrs & $58.57(8.67)$ & $57.28(8.48)$ & $58.26(8.40)$ & $58.96(8.75)$ & $59.84(8.85)$ & $<0.001$ \\
\hline $\mathrm{BMI}$, mean (SD), kg/m² & $23.52(3.83)$ & $23.11(3.50)$ & $23.40(3.93)$ & $23.67(3.79)$ & $23.94(4.04)$ & $<0.001$ \\
\hline Systolic BP, mean (SD), mmHg & $129.10(21.12)$ & $125.91(19.62)$ & $127.53(20.32)$ & $130.25(22.77)$ & $132.93(21.00)$ & $<0.001$ \\
\hline Diastolic BP, mean (SD), mmHg & $75.31(11.90)$ & $73.64(11.05)$ & $74.80(11.84)$ & $75.90(12.31)$ & $77.01(12.12)$ & $<0.001$ \\
\hline Triglycerides, mean (SD), mg/dL & $127.80(91.54)$ & $113.80(75.23)$ & $121.47(83.24)$ & $129.72(93.76)$ & 147.15 (108.29) & $<0.001$ \\
\hline Total cholesterol, mean (SD), mg/dL & $194.14(37.34)$ & $189.61(35.93)$ & $192.91(36.92)$ & $196.06(38.28)$ & $198.22(37.71)$ & $<0.001$ \\
\hline HDL Cholesterol, mean (SD), mg/dL & $51.58(15.18)$ & $52.95(14.23)$ & $52.03(14.67)$ & $51.26(15.88)$ & $50.01(15.76)$ & $<0.001$ \\
\hline LDL Cholesterol, mean (SD), mg/dL & $117.41(34.35)$ & $115.58(32.06)$ & $117.34(32.96)$ & $118.83(35.08)$ & $117.93(37.16)$ & 0.135 \\
\hline Fasting glucose, mean (SD), mg/dL & $108.76(32.34)$ & $110.33(40.72)$ & $108.00(33.38)$ & $108.18(27.66)$ & $108.46(24.90)$ & 0.280 \\
\hline $\mathrm{SCr}$, mean (SD), mg/dL & $0.76(0.16)$ & $0.66(0.12)$ & $0.72(0.13)$ & $0.78(0.14)$ & $0.87(0.15)$ & $<0.001$ \\
\hline eGFR, mean (SD), mL/min per $1.73 \mathrm{~m}^{2}$ & $94.15(12.53)$ & $99.57(10.83)$ & $95.82(11.16)$ & $92.77(12.47)$ & $88.14(12.74)$ & $<0.001$ \\
\hline
\end{tabular}

BMI, body mass index; BP, blood pressure; eGFR, estimated glomerular filtration rate; HDL, high-density lipoprotein; LDL, low-density lipoprotein; SCr, serum creatinine; SUA, serum uric acid; SD, standard deviation; yrs, years

*P value by ANOVA for continuous variables and chi-square test for categorical variables

\section{Discussion}

In this national population-based cohort study in China, we found that higher SUA levels were associated with an increased risk of incident CKD, independent of other risk factors and consistent across subgroups. This positive association was also confirmed by findings in a metaanalysis of 30 cohort studies in multiple populations.

In our study, participants with each $1 \mathrm{mg} / \mathrm{dL}$ increment in the SUA levels had 49\% higher risk of incident CKD during the four-year follow-up, and participants in the fourth SUA quartile were 2.73 times as likely to develop incident CKD compared to those in the first quartile. Our findings were generally consistent with those reported in three previous cohort studies in mainland China [10, $19,20]$. In a community-based cohort study among 1,410 participants aged 40 years or above, the OR of CKD was 1.19 for each $1 \mathrm{mg} / \mathrm{dL}$ increment and 2.14 for the fourth versus first quartile [10], which were equivalent to ours with a similar population-based design. The same study also showed robust associations after excluding participants with hypertension and diabetes. Two other studies in mainland China each using health check-up data for over 5,000 participants from a regional tertiary hospital found $60-70 \%$ higher odds of incident CKD for the extreme-quartile comparison of SUA during a follow-up of 5-6 years $[19,20]$, and one of these two studies showed that the time-mean SUA accounting for fluctuations of SUA could increase the odds of incident CKD by 6.32 fold for the similar comparison [20]. These two hospitalbased studies seemed to observe weaker associations 
Table 2 Association between SUA levels and incident CKD in China

\begin{tabular}{lllll}
\hline & CKD* / no. of participants & $\begin{array}{l}\text { Model 1 } \\
\text { OR (95\% Cl) }\end{array}$ & $\begin{array}{l}\text { Model 2 } \\
\text { OR (95\% Cl) }\end{array}$ & $\begin{array}{l}\text { Model 3 } \\
\text { OR (95\% Cl) }\end{array}$ \\
\hline $\begin{array}{llll}\text { Hyperuricemia }{ }^{\#} \\
\text { No }\end{array}$ & & & 1.00 (reference) & 1.00 (reference) \\
Yes & $156 / 4208$ & 1.00 (reference) & $1.73(0.98-3.06)$ \\
SUA levels & $23 / 338$ & $1.87(1.07-3.27)$ & $1.82(1.04-3.20)$ & 1.00 \\
Quartile 1 & & & 1.00 & $1.44(0.86-2.38)$ \\
Quartile 2 & $27 / 1165$ & 1.00 & $1.44(0.87-2.39)$ & $1.89(1.15-3.10)$ \\
Quartile 3 & $38 / 1133$ & $1.46(0.88-2.41)$ & $1.89(1.16-3.10)$ & $2.73(1.65-4.50)$ \\
Quartile 4 & $49 / 1150$ & $1.93(1.18-3.15)$ & $2.77(1.69-4.55)$ & $P<0.001$ \\
Pfor trend & $66 / 1098$ & $2.80(1.72-4.56)$ & $P<0.001$ & $1.49(1.28-1.74)$ \\
Each 1 mg/dL & & $P<0.001$ & $1.50(1.29-1.74)$ & \\
\hline
\end{tabular}

Model 1: adjusted for age (continuous, years), sex (male and female), residence area (rural and urban), education level (illiterate, primary school, middle school or above). Model 2: adjusted for variables in Model 1 plus body mass index (continuous, $\mathrm{kg} / \mathrm{m}^{2}$ ), smoking status (never, former, and current), and alcohol consumption (never, former, and current); Model 3: adjusted for variables in Model 2 plus systolic BP (continuous, mmHg), total cholesterol (continuous, mg/dL), triglyceride (continuous, $\mathrm{mg} / \mathrm{dL}$ ), and prevalent diabetes mellitus (yes and no)

CKD, chronic kidney disease; $\mathrm{Cl}$, confidence interval; OR, odds ratio; SUA, serum uric acid

* CKD which was defined as eGFR $<60 \mathrm{~mL} / \mathrm{min} / 1.73 \mathrm{~m}^{2}$ based on the Chronic Kidney Disease Epidemiology Collaboration (CKD-EPI)

\# Hyperuricemia was defined as a level of SUA $\geq 7.0 \mathrm{mg} / \mathrm{dL}$ in $\mathrm{men}$ or $\geq 6.0 \mathrm{mg} / \mathrm{dL}$ in women

than those in general populations, possibly due to a narrower spectrum of participants who might have certain medical conditions, and the much stronger association for time-mean SUA might reflect that average increase of SUA could be a more robust predictor of CKD than the increase of a one-time measurement. Of note, another cohort study among 3,605 participants in Chinese Taiwan showed that progressively elevated SUA and persistently high SUA both were associated with two-fold risk of CKD over a 5-year follow-up, compared to those with persistently lower SUA, which further demonstrates the utility of longitudinal SUA change for predicting incident CKD [21]. In addition, our findings based on both the CKD-EPI and MDRD criteria for determining CKD showed consistent positive associations between SUA and CKD, which agreed well with results from other two Chinese studies using these two sets of criteria $[9,19]$. A large retrospective cohort study in over 94,000 Taiwanese participants aged $\geq 20$ years showed that participants in the fifth SUA quintile was associated with a $12-15 \%$ higher risk of incident CKD compared with those in the first SUA quintile during a mean follow-up of 3.5 years [9]. Furthermore, other large cohort studies in non-Chinese populations in Japan [22], Korea [23], US [24], and Austria [25] supported the positive association between SUA and CKD.

However, null associations were also reported between each $1 \mathrm{mg} / \mathrm{dL}$ increment in SUA and incident CKD in a study among 5,888 adults aged $\geq 65$ years in the US (OR, 1.00; 95\% CI, 0.89-1.14) [26] and another study among 800 adults over 65 years in Chinese Taiwan
(1.00; 0.85-1.18) [7], as well as a study in 1,094 employees of an energy generation and distribution company in Brazil (1.12; 0.83-1.50) [27]. These inconsistencies could be potentially explained by age heterogeneity and/or small sample sizes. A previous meta-analysis of 15 cohort studies showed pooled RRs of 1.26 (95\% CI, 1.21-1.31) for each $1 \mathrm{mg} / \mathrm{dL}$ increment in the $<60$ years group versus $1.04(0.96-1.13)$ in the $\geq 60$ years group $(P$ for heterogeneity $=0.004)$, although the number of studies was small in the older age group [6]. Moreover, while there was a lack of statistical significance for the association between hyperuricemia and incident CKD in our Chinese population, it was suggested otherwise in another meta-analysis of 13 cohort studies [28]. These limitations of and uncertainties around previous original and meta-analysis studies demonstrate the necessity to conduct an additional systematic review to update the current evidence.

Our meta-analysis further extended the evidence beyond the Chinese population. Our meta-analysis of 30 studies among 577,334 participants consistently showed that higher SUA was associated with an increased risk of CKD using SUA as continuous, dichotomous, and categorical quartile variables. Our findings were fairly consistent with two previous metaanalyses published five years ago that contained less than half of the data $[6,28]$. Of note, the age heterogeneity for assessing each $1 \mathrm{mg} / \mathrm{dL}$ increment of SUA, which existed in a previous meta-analysis [6], was not observed in the current meta-analysis when more data were available. Given the potential link between SUA 


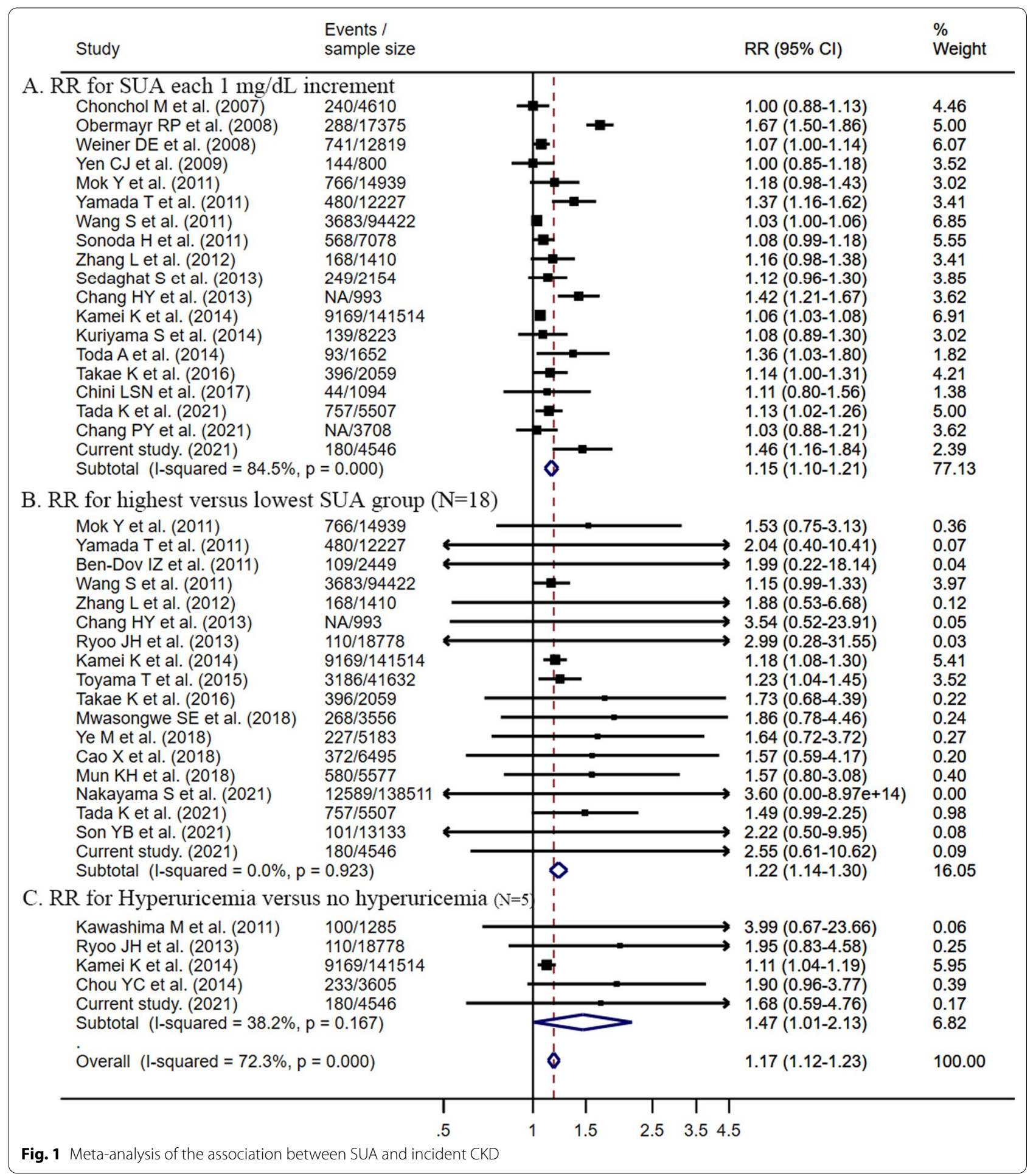

and CKD and high prevalence of hyperuricemia (up to 8.4\% in 2009-2010) in Chinese adults [29], our findings may reinforce the need for timely and routine monitoring of uric acid levels for prevention of incident CKD in clinical practice.
Although the mechanisms underlying the link between SUA and CKD are still uncertain, there have been several lines of evidence. First, uric acid crystals may cause direct damage to the kidney through precipitation in renal tubules [30]. Second, uric acid induces immune system 


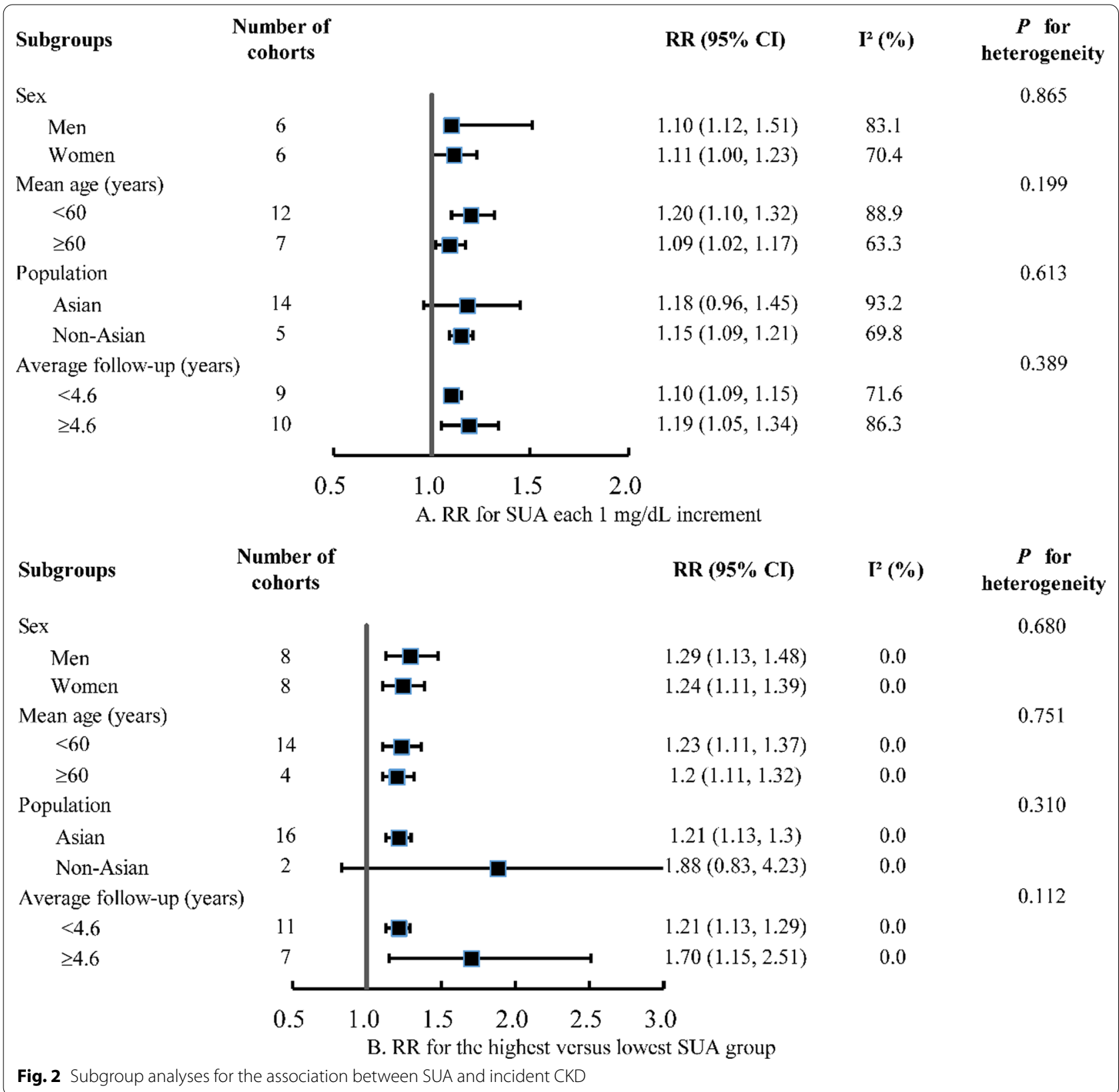

activation and alters the characteristics of resident kidney cells, which contributes to renal inflammation and fibrosis [31]. Third, elevated SUA levels could lead to the development of hypertension that compromises renal function [32]. Fourth, SUA may be a marker of metabolic syndrome and diabetes, which are conventional risk factors for CKD [33]. Fifth, the link between elevated SUA and CKD could be attributable to shared lifestyle risk factors such as unhealthy diet, which could not be fully adjusted for in statistical models.
The current study has major strengths including a large sample size, prospective nature, and a comprehensive meta-analysis. However, several limitations should be acknowledged in our original analyses. First, the study population was part of the CHARLS, and a considerable proportion of participants were excluded due to data missing or loss to follow-up, which may lead to selection and information bias. Second, we could not control for the confounding effects of medications for hyperuricemia such as allopurinol and lifestyle factors such as 
dietary and physical activity due to data unavailability, and residual confounding was thus likely to exist. Third, the follow-up lasted only 4 years and reverse causality could not be ruled out, i.e., CKD might lead to increased SUA instead of the other way around. However, our meta-analysis consistently confirmed the positive association in subgroups of studies with different follow-up durations. Fourth, we did not have repeated measurements of SUA before CKD ascertainment and could not account for its fluctuation in our analyses. However, our sensitivity analysis using a time-mean measure of SUA supported our overall findings.

\section{Conclusion}

Higher SUA levels were associated with an increased risk of incident CKD in Chinese middle-aged and elderly adults. Such a positive association was supported by findings from a meta-analysis across multiple populations. Given the high prevalence of hyperuricemia in the general population, it is imperative to understand whether reducing SUA levels could lead to lower CKD risk. Future clinical trials and mechanistic studies may improve our understanding of the link between SUA and CKD and inform potential interventions in the population.

\section{Abbreviations \\ BMI: Body mass index; BP: Blood pressure; CHARLS: China Health and Retire- ment Longitudinal Study; CKD: Chronic kidney disease; Cl: Confidence interval; eGFR: Estimated glomerular filtration rate; FBG: Fasting blood glucose; HbA1c: Glycosylated hemoglobin; HDL: High-density lipoprotein; HR: Hazard ratio; LDL: Low-density lipoprotein; OR: Odds ratio; RR: Relative risk; SD: Standard deviation; SUA: Serum uric acid; TC: Total cholesterol; TG: Triglyceride.}

\section{Supplementary Information}

The online version contains supplementary material available at https://doi. org/10.1186/s12986-021-00618-4.

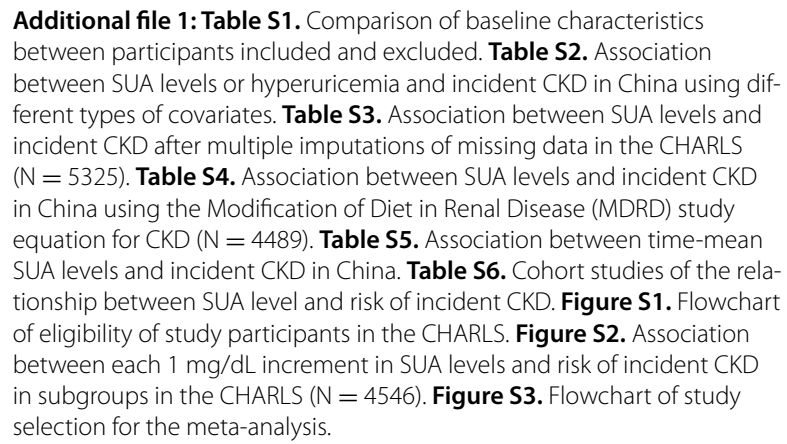

Additional file 1: Table S1. Comparison of baseline characteristics between participants included and excluded. Table S2. Association between SUA levels or hyperuricemia and incident CKD in China using different types of covariates. Table S3. Association between SUA levels and incident CKD after multiple imputations of missing data in the CHARLS ( $N=5325$ ). Table S4. Association between SUA levels and incident CKD in China using the Modification of Diet in Renal Disease (MDRD) study equation for CKD ( $N=4489)$. Table S5. Association between time-mean SUA levels and incident CKD in China. Table S6. Cohort studies of the relationship between SUA level and risk of incident CKD. Figure S1. Flowchart of eligibility of study participants in the CHARLS. Figure S2. Association between each $1 \mathrm{mg} / \mathrm{dL}$ increment in SUA levels and risk of incident CKD in subgroups in the CHARLS (N = 4546). Figure S3. Flowchart of study selection for the meta-analysis.

\section{Acknowledgements}

We thank the China Center for Economic Research, National School of Development at Peking University for providing the CHARLS data.

\section{Authors' contributions}

NW, YL, and XFP designed the study. NW and JX analyzed the data. NW drafted the first manuscript. JX, SC, CY, YX, CX, TY, NL, YL, and XFP provided critical revision of the manuscript for important intellectual content. All authors contributed to the interpretation of the data and approved the final version of the manuscript. All authors read and approved the final manuscript.

\section{Funding}

XF Pan was supported by the International Postdoctoral Exchange Fellowship Program of the China Postdoctoral Council (No 20180062). The study was supported by the Chengdu Development Program for Excellent Clinical Specialties. The funding agencies had no role in design, analysis, interpretation, or writing of this study.

\section{Availability of data and materials}

The datasets used during the current study are available in the CHARLS official website, http://charls.pku.edu.cn/. All data, analytic methods, and study materials presented within this article are available from the corresponding author on reasonable request.

\section{Declarations}

\section{Ethics approval and consent to participate}

The study was approved by the Biomedical Ethics Review Committee of Peking University (IRB00001052-11015), and informed consent was obtained from all participants.

\section{Consent for publication}

Not applicable.

\section{Competing interests}

The authors declare that they have no competing interests.

\section{Author details}

${ }^{1}$ Department of Health and Social Behavior, West China School of Public Health and West China Fourth Hospital, Sichuan University, Chengdu, Sichuan, China. ${ }^{2}$ Kidney Disease Center, The First Affiliated Hospital, College of Medicine, Zhejiang University, Hangzhou, Zhejiang, China. ${ }^{3}$ Department of Gastroenterology, The First Affiliated Hospital, College of Medicine, Zhejiang University, Hangzhou, Zhejiang, China. ${ }^{4}$ Center for Obesity and Metabolic Health, The Third People's Hospital of Chengdu \& The Affiliated Hospital of Southwest Jiaotong University, Chengdu, Sichuan, China. ${ }^{5}$ Center of Gastrointestinal and Minimally Invasive Surgery, Department of General Surgery, The Third People's Hospital of Chengdu \& The Affiliated Hospital of Southwest Jiaotong University, Chengdu, Sichuan, China. ${ }^{6}$ Division of Epidemiology, Department of Medicine, Vanderbilt Epidemiology Center, Vanderbilt University Medical Center, Nashville, TN, USA. ${ }^{7}$ Department of Epidemiology and Biostatistics, and Ministry of Education Key Lab of Environment and Health, School of Public Health, Tongji Medical College, Huazhong University of Science and Technology, Wuhan, Hubei, China. ${ }^{8}$ The George Institute for Global Health, Faculty of Medicine, University of New South Wales, Sydney, NSW, Australia.

Received: 22 June 2021 Accepted: 25 September 2021

Published online: 19 October 2021

\section{References}

1. GBD Chronic Kidney Disease Collaboration. Global, regional, and national burden of chronic kidney disease, 1990-2017: a systematic analysis for the Global Burden of Disease Study 2017. Lancet. 2020;395(10225):709-33.

2. Matsushita K, van der Velde M, Astor BC, et al. Association of estimated glomerular filtration rate and albuminuria with all-cause and cardiovascular mortality in general population cohorts: a collaborative meta-analysis. Lancet. 2010;375(9731):2073-81.

3. Webster AC, Nagler EV, Morton RL, Masson P. Chronic kidney disease. Lancet. 2017;389(10075):1238-52. 
4. Johnson RJ, Nakagawa T, Jalal D, Sánchez-Lozada LG, Kang DH, Ritz E. Uric acid and chronic kidney disease: which is chasing which? Nephrol Dial Transplant. 2013;28(9):2221-8.

5. Xia X, Luo Q, Li B, Lin Z, Yu X, Huang F. Serum uric acid and mortality in chronic kidney disease: a systematic review and meta-analysis. Metabolism. 2016;65(9):1326-41.

6. Zhu P, Liu Y, Han L, Xu G, Ran JM. Serum uric acid is associated with incident chronic kidney disease in middle-aged populations: a meta-analysis of 15 cohort studies. PLoS ONE. 2014;9(6):e100801.

7. Yen CJ, Chiang CK, Ho LC, et al. Hyperuricemia associated with rapid renal function decline in elderly Taiwanese subjects. J Formos Med Assoc. 2009;108(12):921-8.

8. Chien KL, Lin HJ, Lee BC, Hsu HC, Lee YT, Chen MF. A prediction model for the risk of incident chronic kidney disease. Am J Med. 2010;123(9):836-46

9. Wang S, Shu Z, Tao Q, Yu C, Zhan S, Li L. Uric acid and incident chronic kidney disease in a large health check-up population in Taiwan. Nephrology (Carlton). 2011;16(8):767-76.

10. Zhang L, Wang F, Wang $X$, Liu L, Wang $H$. The association between plasma uric acid and renal function decline in a Chinese population-based cohort. Nephrol Dial Transplant. 2011;27(5):1836-9.

11. Zhao Y, Hu Y, Smith JP, Strauss J, Yang G. Cohort profile: the China Health and Retirement Longitudinal Study (CHARLS). Int J Epidemiol. 2014:43(1):61-8.

12. Roubenoff R. Gout and hyperuricemia. Rheum Dis Clin N Am. 1990;16(3):539-50.

13. Levey AS, Stevens LA, Schmid $\mathrm{CH}$, et al. A new equation to estimate glomerular filtration rate. Ann Intern Med. 2009;150(9):604-12.

14. Stevens LA, Coresh J, Greene T, Levey AS. Assessing kidney function-measured and estimated glomerular filtration rate. N Engl J Med. 2006:354(23):2473-83.

15. Liu LS. Writing Group of 2010 Chinese Guidelines for the Management of Hypertension. 2010 Chinese guidelines for the management of hypertension. Zhonghua Xin Xue Guan Bing Za Zhi. 2011;39(7):579-615.

16. American Diabetes Association. Classification and diagnosis of diabetes: standards of medical care in diabetes - 2020. Diabetes Care. 2020;43(Suppl 1):S14-31.

17. Joint Committee for Developing Chinese guidelines on Prevention and Treatment of Dyslipidemia in Adults. Chinese guidelines on prevention and treatment of dyslipidemia in adults. Zhonghua Xin Xue Guan Bing Za Zhi. 2007;35(5):390-419.

18. Pan XF, Wang L, Pan A. Epidemiology and determinants of obesity in China. Lancet Diabetes Endocrinol. 2021;9(6):373-92.

19. Cao X, Wu L, Chen Z. The association between elevated serum uric acid level and an increased risk of renal function decline in a health checkup cohort in China. Int Urol Nephrol. 2018;50(3):517-25.
20. Ye M HuK Jin J, Wu D Hu P He Q The association between time-mean serum uric acid levels and the incidence of chronic kidney disease in the general population: a retrospective study. BMC Nephrol. 2018;19(1):190.

21. Chou YC, Kuan JC, Yang T, et al. Elevated uric acid level as a significant predictor of chronic kidney disease: a cohort study with repeated measurements. J Nephrol. 2015;28(4):457-62.

22. Toyama T, Furuichi $K$, Shimizu M, et al. Relationship between serum uric acid levels and chronic kidney disease in a Japanese cohort with normal or mildly reduced kidney function. PLoS ONE. 2015;10(9):e0137449.

23. Ryoo JH, Choi JM, Oh CM, Kim MG. The association between uric acid and chronic kidney disease in Korean men: a 4-year follow-up study. J Korean Med Sci. 2013;28(6):855-60.

24. Weiner DE, Tighiouart H, Elsayed EF, Griffith $J$ L, Salem DN, Levey AS. Uric acid and incident kidney disease in the community. J Am Soc Nephrol. 2008;19(6):1204-11

25. Obermayr RP, Temml C, Knechtelsdorfer M, Gutjahr G, Kletzmayr J, Heiss $\mathrm{S}$, et al. Predictors of new-onset decline in kidney function in a general middle-european population: nephrology dialysis transplantation. Nephrol Dial Transplant. 2008;23(4):1265-73.

26. Chonchol M, Shlipak MG, Katz R, et al. Relationship of uric acid with progression of kidney disease. Am J Kidney Dis. 2007;50(2):239-47.

27. Chini LSN, Assis LIS, Lugon JR. Relationship between uric acid levels and risk of chronic kidney disease in a retrospective cohort of Brazilian workers. Braz J Med Biol Res. 2017;50(9):e6048.

28. Li L, Yang C, Zhao Y, Zeng X, Liu F, Fu P. Is hyperuricemia an independent risk factor for new-onset chronic kidney disease?: a systematic review and metaanalysis based on observational cohort studies. BMC Nephrol. 2014;15:122.

29. Liu $H$, Zhang XM, Wang YL, Liu BC. Prevalence of hyperuricemia among Chinese adults: a national cross-sectional survey using multistage, stratified sampling. J Nephrol. 2014;27(6):653-8.

30. Hahn K, Kanbay M, Lanaspa MA, Johnson RJ, Ejaz AA. Serum uric acid and acute kidney injury: a mini review. J Adv Res. 2017;8(5):529-36.

31. Jung SW, Kim SM, Kim YG, Lee SH, Moon JY. Uric acid and inflammation in kidney disease. Am J Physiol Renal Physiol. 2020;318(6):F1327-40.

32. Soltani Z, Rasheed K, Kapusta DR, Reisin E. Potential role of uric acid in metabolic syndrome, hypertension, kidney injury, and cardiovascular diseases: is it time for reappraisal? Curr Hypertens Rep. 2013;15(3):175-81.

33. Cirillo P, Sato W, Reungjui S, et al. Uric acid, the metabolic syndrome, and renal disease. J Am Soc Nephrol. 2006;17(12 Suppl 3):S165-8.

\section{Publisher's Note}

Springer Nature remains neutral with regard to jurisdictional claims in published maps and institutional affiliations.
Ready to submit your research? Choose BMC and benefit from:

- fast, convenient online submission

- thorough peer review by experienced researchers in your field

- rapid publication on acceptance

- support for research data, including large and complex data types

- gold Open Access which fosters wider collaboration and increased citations

- maximum visibility for your research: over $100 \mathrm{M}$ website views per year

At $\mathrm{BMC}$, research is always in progress.

Learn more biomedcentral.com/submissions 